\title{
Autism Spectrum: Impact of Diagnosis on Children's Parents
}

\author{
Rosa Martins ${ }^{1}$, Nélia Carvalho ${ }^{1}$, Ana Andrade Conceição Martins ${ }^{1}$ Sofia Campos ${ }^{1}$
}

\section{1-Escola Superior de Saúde do Instituto Politécnico de Viseu- Portugal}

\begin{abstract}
When a child with autism is born, the impact of the diagnosis on the parents causes the family life cycle to change, requiring an additional effort to reorganize the new family. Thus, it is essential to evaluate this impact on Parents, as well as the factors associated with them.

Non-experimental, cross-sectional, descriptive-correlational and quantitative study. Used a non-probabilistic convenience sample consisting of 138 parents with autistic children mostly female $(67.1 \%)$ with mean age of 42.32 years. In the data collection, a questionnaire was used with sociodemographic data (relative to parents and children), neonatal data and informational context, and an evaluation scale of the impact of the diagnosis of autism in the parents (EAIDAP) of Mónica Febra, 2009.

The data show that the impact of the diagnosis of autism on parents was moderate. Parents show great concern about the future of their children, they think that they have not yet been able to completely overcome the diagnostic shock and present less difficulties in adapting their social characteristics. The impact of the diagnosis was higher in parents who lived risk pregnancies, with dystocia (not normal) and were alone when they learned of the diagnosis.

The conclusions of this study point to the promotion of intervention strategies by health professionals in order to minimize the negative impact that this diagnosis causes in families
\end{abstract}

Keywords: Autism; Parents; Impact of Diagnosis, Families. 
